\title{
PERCEPÇÃO DA QUALIDADE DE VIDA POR PESSOAS COM DEFICIÊNCIAS FÍSICAS PRATICANTES E NÃO PRATICANTES DE ATIVIDADES DE LAZER
}

Recebido em: 15/04/2012

Aceito em: 21/11/2012

Rejane Martins Canedo Lima Universidade de Franca (UNIFRAN)

Franca - SP - Brasil

Priscilla Rosa Queiroz Ribeiro

Universidade Federal de Uberlândia - UFU

Uberlândia - MG - Brasil

Maria Georgina Marques Tonello Universidade de Franca (UNIFRAN)

Franca - SP - Brasil

RESUMO: O objetivo desse estudo foi verificar a percepção que as pessoas com deficiências físicas praticantes e não praticantes de atividades de lazer possuem da sua qualidade de vida. Participaram deste estudo 100 pessoas com deficiências físicas, sendo $45 \%$ do sexo feminino e $55 \%$ do sexo masculino, com idade média de 42,06 $\pm 13,80$ anos da cidade de Patos de Minas MG. A amostra foi avaliada através de um questionário com informações gerais, abordando a participação em atividades de lazer. Para avaliar a percepção da qualidade de vida foi aplicado o questionário WHOQOL breve. Os participantes que realizavam atividades de lazer apresentaram diferenças estatísticas significantes, em todos os domínios de qualidade de vida: físico, psicológico, relações sociais e meio ambiente. Podemos concluir que as atividades de lazer apresentaram uma grande importância na percepção de qualidade de vida dos entrevistados.

PALAVRAS CHAVE: Qualidade de Vida. Atividades de Lazer. Pessoas com Deficiência.

\section{PERCEPTION OF QUALITY OF LIFE FOR DISABLED PRACTITIONERS AND NON PRACTITIONERS OF LEISURE ACTIVITIES}

ABSTRACT: The aim of this study was to assess the perception that people with disabilities and non-practitioners engaged in recreation have on their quality of life. The study included 100 people with physical disabilities, $45 \%$ female and 55\% male, mean age $42.06 \pm 13.80$ city of Patos de Minas - MG. The sample was assessed using a questionnaire with general information, addressing participation in leisure activities. To assess the perception of quality of life of the sample was applied the WHOQOL - brief. Participants who performed leisure activities showed statistically significant differences 
in all domains of quality of life: physical, psychological, social relationships and environment. We can conclude that leisure activities have great importance in the perception of quality of life of the respondents.

KEYWORDS: Quality of Life. Leisure Activities. Disabled Persons.

\section{INTRODUÇÃO}

Com a nova visão de saúde advinda do conceito mais amplo atribuído a qualidade de vida pela Organização Mundial de Saúde, como não apenas a ausência de doenças, mas sim um estado de bem-estar físico, mental e social, ganharam espaço as políticas de promoção de saúde com vistas a melhorar, através dessas políticas e as diretrizes por elas estabelecidas, os níveis de saúde da população e, consequentemente, a sua qualidade de vida.

A Organização Mundial de Saúde (THE WHOQOL GROUP, 1995) conceitua qualidade de vida como a percepção do indivíduo em relação a sua posição na vida, no contexto da cultura e do sistema de valores nos quais vive e em relação aos seus objetivos, expectativas, padrões e preocupações. Envolve o bem estar físico, mental, psicológico e emocional, além de relacionamentos sociais, como família e amigos e também a saúde, educação, poder de compra e outras circunstâncias da vida.

Com o crescimento cada vez maior da preocupação com a inserção social de pessoas com deficiência, ganharam espaço também as preocupações relativas à qualidade de vida dessa população. Segundo Diehl (2008), a deficiência física pode ser caracterizada por um comprometimento físico que restringe não apenas os movimentos de um indivíduo, mas também seu convívio social. Além das barreiras arquitetônicas que impedem seu acesso aos espaços sociais ou de trabalho, existe a barreira presente no discurso revestido de preconceitos e mitos das pessoas. Como por exemplo, o estereótipo construído pela mídia de "corpo padrão", sendo o corpo "belo" e "perfeito", 
o que automaticamente exclui as pessoas com deficiência física, visto que para a grande maioria ser bonito é ser "normal". Consequentemente, Gorgatti e Gorgatti (2008) mencionam que muitas pessoas com deficiência física tendem a se sentirem feias, pouco atraentes e extremamente limitadas.

Nesse contexto de busca pela melhora na qualidade de vida, a prática de lazer com o intuito de promoção de saúde tem sido destaque nos últimos anos em toda a mídia. Nunca se falou tanto sobre os efeitos benéficos e da prática adequada de lazer como condição essencial para a manutenção de condições positivas de saúde e melhora da qualidade de vida, especialmente em pessoas com deficiência. Shephard (1991) ressalta que se a percepção sobre a saúde há maior probabilidade de aumentar a produtividade de indivíduos com deficiência física. Assim, o investimento em programas de lazer que promovam a saúde para essa população tem o potencial de gerar menor custo para o indivíduo, para a sociedade e para a saúde pública.

De acordo com Noce, Simim e Mello (2009) pessoas com deficiências físicas ou de qualquer outra deficiência obtêm efeitos positivos para a saúde física, mental e social pela prática regular de atividades de lazer ou desportivas adaptadas, tenham estas finalidades competitivas ou não..

Tendo em vista a crescente importância da abordagem da qualidade de vida das pessoas com deficiência e dos vários fatores relacionados a ela o objetivo desse estudo foi verificar a percepção que as pessoas com deficiências físicas praticantes e não praticantes de lazer da cidade de Patos de Minas - MG possuem acerca da sua qualidade de vida. 


\section{MATERIAL E MÉTODOS}

\subsection{Participantes}

Participaram deste estudo 100 pessoas com deficiências físicas, sendo $45 \%$ do sexo feminino e 55\% do sexo masculino, com idade média de 42,06 $\pm 13,80$ anos, selecionadas a partir de um levantamento de todas as pessoas com deficiências físicas na ADEFIPAM - Associação dos Deficientes Físicos de Patos de Minas MG e que se prontificaram a participar da pesquisa e assinaram o Termo de Consentimento Livre e Esclarecido com esclarecimentos sobre os objetivos do estudo, procedimentos, possíveis riscos, desconfortos e benefícios.

Este projeto foi submetido ao Comitê de Ética em Pesquisa da Universidade de Franca - UNIFRAN, através do processo número 0006/10, obedecendo às normas internacionais e legislação nacional vigentes para realização de pesquisa envolvendo seres humanos e teve o parecer favorável para sua realização, de acordo com a resolução 196/96, que regulamenta pesquisa com seres humanos.

\subsection{Instrumentos e procedimentos}

Foi feita uma caracterização dos participantes, através de uma ficha que constou das seguintes perguntas: idade, sexo, tipo de deficiência, renda, nível de instrução, condições de moradia e transporte e atividades físicas e de lazer que participam.

Para avaliar a percepção da qualidade de vida da amostra foi aplicado o questionário WHOQOL - breve contendo 26 perguntas, das quais 24 são distribuídas em quatro domínios: físico, psicológico, relações sociais e meio-ambiente. Os domínios, representados por facetas, são constituídos por questões formuladas para uma escala de 
respostas do tipo Likert, com escala de intensidade (nada-extremamente), capacidade (nada-completamente), frequência (nunca-sempre) e avaliação (muito insatisfeito-muito satisfeito; muito ruim-muito bom) (OMS, 1998) .Para cada aspecto da qualidade de vida expresso no questionário WHOQOL-breve, o sujeito pode apresentar sua resposta por meio de escores que variam de um a cinco, sendo a condição pior no escore expressa pelo valor um e a melhor pelo valor cinco. Os resultados dos domínios apresentam valores entre zero e cem, sendo piores os mais próximos de zero e melhores os mais próximos de cem. Dessa forma, um sujeito que apresente valor igual a 50 para determinado domínio pode ser considerado mediano para esse domínio.

\section{3. Análise estatística}

Para a análise dos dados do questionário sócio-demográfico foi utilizada estatística descritiva composta por média, desvio padrão e distribuição de freqüência. Para análise estatística dos dados do questionário de qualidade de vida - WHOQOLbreve foram utilizadas as sintaxes disponíveis e recomendadas para o programa estatístico SPSS versão 13, favorecendo a análise dos domínios.

Para fazer a comparação entre os grupos foi utilizado o teste t para amostra independente. O teste Mann-Whitney somente foi aplicado para variáveis que não atenderam o pressuposto da distribuição normal dos dados exigidos na utilização do teste $\mathrm{t}$ student. 


\section{RESULTADOS}

As características da amostra do presente estudo estão apresentadas na (TAB. 1).

TABELA 1

Caracterização da amostra.

\begin{tabular}{|c|c|c|}
\hline & Categorias & Percentual $(\%)$ \\
\hline \multirow{2}{*}{ Sexo } & Masculino & 55 \\
\hline & Feminino & 45 \\
\hline \multirow{5}{*}{ Faixa Etária } & 18 a 25 anos & 14 \\
\hline & 26 a 35 anos & 23 \\
\hline & 36 a 45 anos & 25 \\
\hline & 46 a 55 anos & 21 \\
\hline & Acima de 56 anos & 17 \\
\hline \multirow{5}{*}{ Estado civil } & Casado & 32 \\
\hline & Amasiado & 5 \\
\hline & Solteiro & 46 \\
\hline & Viúvo & 6 \\
\hline & Divorciado & 11 \\
\hline \multirow{6}{*}{ Grau de instrução } & $1^{\circ}$ Grau incompleto & 39 \\
\hline & $1^{\circ}$ Grau completo & 11 \\
\hline & $2^{\circ}$ Grau incompleto & 7 \\
\hline & $2^{\circ}$ Grau completo & 18 \\
\hline & Superior incompleto & 17 \\
\hline & Superior completo & 8 \\
\hline \multirow{5}{*}{ Renda } & Menos de 1 salário mínimo & 1 \\
\hline & 1 a 3 salários mínimos & 78 \\
\hline & 3 a 5 salários mínimos & 11 \\
\hline & 5 a 8 salários mínimos & 8 \\
\hline & Acima de 8 salários mínimos & 2 \\
\hline
\end{tabular}

Fonte: Pesquisa de campo (2010).

Os dados relativos aos tipos de deficiência apresentados pela amostra estão apresentados na (TAB. 2).

TABELA 2

Tipos de deficiências físicas.

\begin{tabular}{|c|c|}
\hline Tipos de deficiência & Percentual (\%) \\
\hline Paralisia infantil & 29 \\
\hline Lesão Medular & 15 \\
\hline Traumatismo Craniano Encefálico & 2 \\
\hline Acidente Vascular Cerebral & 7 \\
\hline Amputação & 10 \\
\hline Paralisia Cerebral & 9 \\
\hline Osteoartrose & 10 \\
\hline Má formação Congênita & 6 \\
\hline Outras & 12 \\
\hline
\end{tabular}

Fonte: Pesquisa de campo (2010). 
A avaliação da qualidade de vida dos integrantes da amostra através do questionário WHOQOL-breve está apresentada na (TAB. 3).

TABELA 3

Média e desvio padrão dos domínios de qualidade de vida do WHOQOL-breve.

\begin{tabular}{c|c|c}
\hline $\begin{array}{c}\text { Domínio da qualidade de vida } \\
\text { (WHOQOL/breve) }\end{array}$ & Média & Desvio Padrão \\
\hline Físico & 65,40 & $\pm 16,80$ \\
Psicológico & 75,78 & $\pm 15,18$ \\
Social & 75,08 & $\pm 21,07$ \\
Ambiente & 62,51 & $\pm 16,21$ \\
\hline
\end{tabular}

Fonte: Pesquisa de campo (2010).

A avaliação da qualidade de vida dos integrantes da amostra através do questionário WHOQOL-breve está apresentada na FIG. 1.

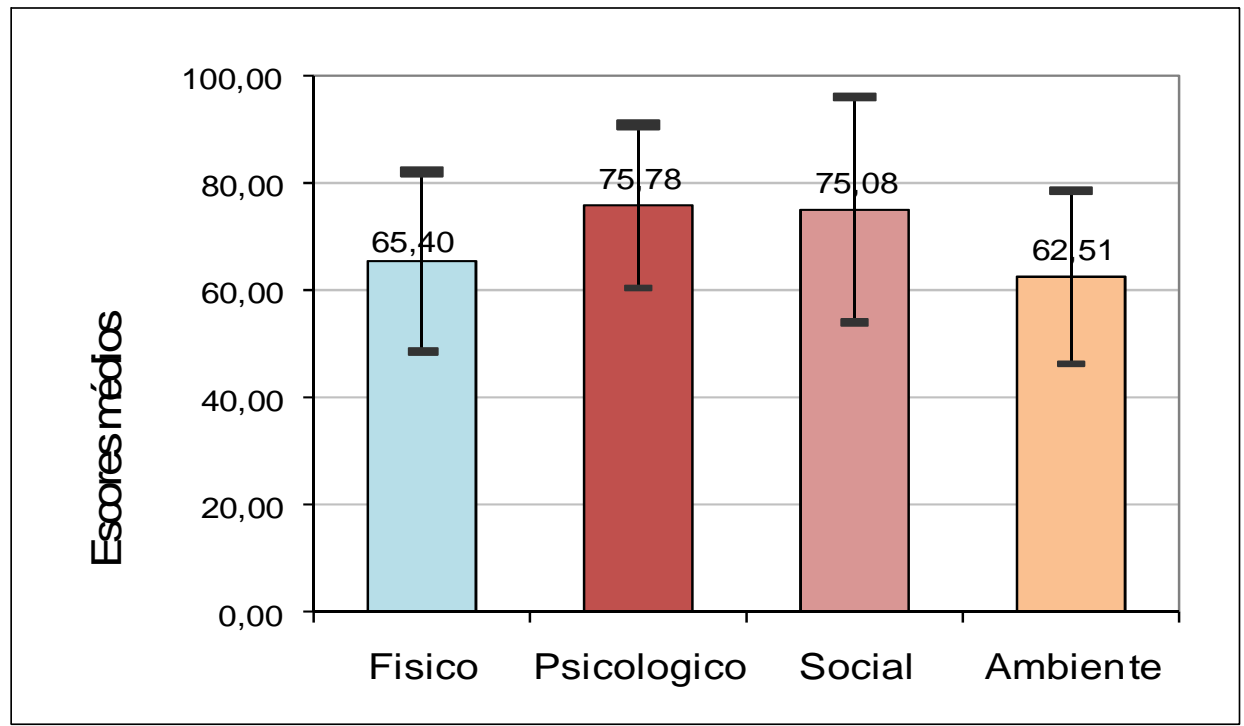

FIGURA 1 - Escores dos domínios de qualidade de vida do WHOQOL-breve.

Fonte: Pesquisa de campo (2010). 
Em relação à participação em atividades de lazer os resultados estão apresentados na TAB. 4. Nota-se que quase a metade da população estudada não participa de nenhuma atividade de lazer (valor percentual 40, percentual acumulado $46,3)$.

TABELA 4

Participação em atividades de lazer

\begin{tabular}{c|c|c|c}
\hline & Frequência (n) & Percentual (\%) & $\begin{array}{c}\text { Percentual } \\
\text { acumulado (\%) }\end{array}$ \\
\hline Teatro & 1 & 0,9 & 1,1 \\
Cinema & 9 & 8,2 & 9,5 \\
Aulas música & 4 & 3,6 & 4,2 \\
Bares e restaurantes & 30 & 27,3 & 31,6 \\
Outros & 22 & 20 & 23,2 \\
Não participo & 44 & 40 & 46,3 \\
Total & $\mathbf{1 1 0}$ & $\mathbf{1 0 0}$ & $\mathbf{1 1 5 , 8}$ \\
\hline
\end{tabular}

Fonte: Pesquisa de campo (2010)

A TAB. 5. apresenta a comparação dos escores obtidos entre os participantes e não participantes de atividades de lazer no questionário de avaliação da qualidade de vida (WHOQOL-breve) nos quatro domínios do questionário.

TABELA 5

Comparação da qualidade de vida de participantes e não participantes de atividades de lazer

\begin{tabular}{lcc|c}
\hline & \multicolumn{2}{c}{$\begin{array}{c}\text { Você participa de alguma atividade de lazer } \\
\text { semanalmente? }\end{array}$} & \\
\hline $\begin{array}{l}\text { Domínio da qualidade de } \\
\text { vida (WHOQOL/breve) }\end{array}$ & $\operatorname{Sim}(\mathrm{n}=51)$ & Não (n=49) & $\mathrm{P}$ \\
Físico & $71,43 \pm 13,94$ & $59,00 \pm 17,34$ & $\mathbf{0 , 0 0 0}$ \\
Psicológico & $81,18 \pm 10,97$ & $70,05 \pm 17,35$ & $\mathbf{0 , 0 0 0}$ \\
Social & $82,52 \pm 13,97$ & $67,35 \pm 24,34$ & $\mathbf{0 , 0 0 0}$ \\
Ambiente & $67,78 \pm 14,36$ & $57,02 \pm 16,33$ & $\mathbf{0 , 0 0 1}$ \\
\hline
\end{tabular}

Fonte: Pesquisa de campo (2010)

A FIG. 2. apresenta a comparação dos escores obtidos entre os participantes e não participantes de atividades de lazer no questionário de avaliação da qualidade de vida (WHOQOL-breve) nos quatro domínios do questionário. 


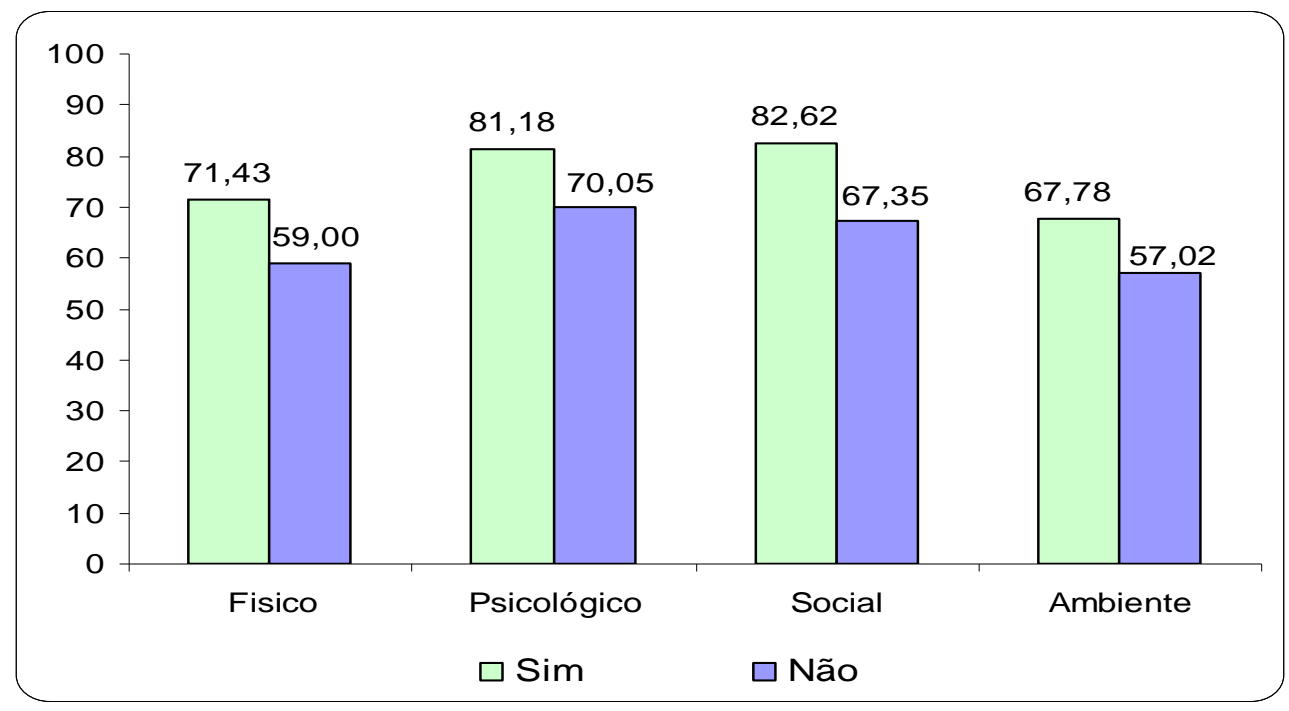

FIGURA 2 - Comparação da qualidade de vida de participantes e não participantes de atividades de lazer.

* Diferença estatisticamente significante entre os participantes e não participantes de atividades de lazer. $\mathrm{P} \leq 0,005$.

Fonte: Pesquisa de campo (2010).

\section{DISCUSSÃO}

Em concordância com os achados do estudo realizado por Noce, Simim e Melo (2009) o tipo de deficiência mais encontrado foi a paralisia infantil, o que pode ter relação com a faixa etária predominantemente apresentada pela amostra do presente estudo (média de 42,06 anos), semelhante também a média de idade apresentada pelos voluntários do estudo dos autores citados anteriormente (38,1 anos).

Quanto a utilização do questionário WHOQOL-breve como meio para avaliar a qualidade de vida Pereira (2006) afirma que a construção do instrumento não prevê um escore específico para avaliar a qualidade de vida como positiva ou negativa. Não existem pontos de corte sobre o qual se possa avaliar a qualidade de vida como "ruim" ou "boa". Eles se configuram numa escala positiva, ou seja, quanto maior o escore, melhor a qualidade de vida. Maiores valores e por consequência maior nível de qualidade de vida foram encontrados nos domínios psicológico e social e menores valores nos domínios físico e ambiente, respectivamente. 
Esses resultados estão em concordância com os resultados encontrados por Bamp, Guilhem e Lima (2008) que em estudo realizado com 111 lesados medulares traumáticos encontraram também piores escores em relação aos domínios ambiente e físico e maiores nos domínios psicológico e social. Acredita-se que os escores mais baixos encontrados nos domínios físico e ambiental em relação ao psicológico e social possa estar associado ao baixo nível de vivência física apresentado por essas pessoas, o que pode exacerbar a dependência física e capacidade funcional e consequentemente causar prejuízos quanto a percepção da qualidade de vida e as inadequadas condições ambientais que ainda se configuram como um fator que gera dificuldades para o dia-adia da pessoa com deficiência.

Ainda que seja conhecida a necessidade de incentivar e estimular as pessoas com deficiência a participarem de programas de lazer, são muitas as dificuldades para a adoção de hábitos ativos por parte dessas pessoas. Acredita-se que a inatividade apresentada pelas pessoas com deficiência da cidade de Patos de Minas possa ser explicada pela escassez de oportunidades oferecidas a essas pessoas para a prática dessas atividades na cidade.

Para Guiselini (1996) ao participar de um programa cuja meta é melhorar a sua qualidade de vida, ajuda a controlar o estresse e reduz a tendência à depressão, permite sensação de mais energia, auxilia na realização das atividades diárias, ajuda a dormir melhor e contribui na estruturação da auto-imagem.

Estudo realizado por Zuchetto e Castro (2002) mostrou que os principais motivos que levam as pessoas com deficiência a praticar atividades são porque o próprio pessoa com deficiência acha importante, por indicação médica, porque a família acha importante e para manter-se ocupado. Este mesmo estudo procurou demonstrar 
também as principais barreiras impostas às pessoas com deficiência para a prática de atividades, o que pode, em parte, explicar o baixo número de pessoas com deficiência que praticam atividades físicas e de lazer a despeito dos benefícios dessa prática, como o encontrado no presente estudo. Entre as dificuldades encontradas as barreiras arquitetônicas e a falta de transporte adaptado foram as de maior ocorrência com $39 \%$ cada. A dificuldade econômica em segundo lugar, com 33\% do total. A falta de oportunidade para a prática de atividades físicas com $22 \%$ e a disponibilidade de tempo, falta de locais e equipamentos adaptados, e nenhuma dificuldade com $6 \%$ das respostas da amostra.

Em relação ao lazer das pessoas com deficiência física, Hunger, Squarcini e Pereira (2004) afirmam “que as condições individuais são responsáveis por barreiras no acesso à prática do lazer, sendo as pessoas portadoras de deficiência as mais afetadas, dadas as limitações física, econômica, cultural, etária, entre outras”.

Segundo a Associação Brasileira de Normas Técnicas (ABNT, 2004), a acessibilidade é definida, na norma NBR 9050/94, como a possibilidade e condição de alcance para utilização, com segurança e autonomia, de edificações, espaço, mobiliário e equipamento urbano.

Nesse sentido, acredita-se que o baixo nível de participação em atividades de lazer encontrado pode ser explicado pela falta de acesso dessa população a essas atividades. De acordo com Hunger, Squarcini e Pereira (2004) "no Brasil ainda há muitas dificuldades em encontrar locais com infra-estrutura adequada".

Os indivíduos que participam de atividades de lazer apresentaram escores mais altos, com diferença estatisticamente significante em todos os domínios do questionário. Estudo realizado por Zuchetto e Castro (2002) mostrou que as atividades 
de lazer que as pessoas com deficiência mais participam são em bares e restaurantes, em casa, na rua ou bairro e cinema. O presente estudo encontrou resultados semelhantes com o percentual mais elevado nas atividades realizadas em bares, restaurantes e cinema. Observa-se nesses estudos a baixa participação em atividades de lazer por parte das pessoas com deficiência e, sobretudo, a pouca diversidade dessa prática. Zuchetto e Castro (2002) afirmam que este fato deve-se em grande parte às barreiras arquitetônicas que dificulta o acesso destas pessoas para os locais citados, outro fator determinante foi a questão econômica, sair de casa custa caro.

A importância do lazer para a pessoa portadora de deficiência física é também destacada em pesquisa realizada por Kinney e Coyle (1992), na qual se entrevistaram 790 pessoas com deficiências adultos. Entre eles, 42\% visam o lazer como fonte para obter uma vida satisfatória e 11\% apresentam outras variáveis, como status financeiro, autoestima, boa saúde, religião e casamento. A pesquisa explicita que o lazer e o nível de vida satisfatório são influenciados pelo fator emprego e se a deficiência foi ou não adquirida. Finalizando, concluem que o lazer aumenta a qualidade de vida da pessoa com deficiência física praticante.

Nesse sentido, nota-se a importância da participação em atividades de lazer por parte de pessoas com deficiência, uma vez que esses fatores influenciam positivamente em sua qualidade de vida.

\section{CONCLUSÃO}

A avaliação da percepção da qualidade de vida das pessoas com deficiência física da cidade de Patos de Minas - MG, obtidas pelo WHOQOL-breve demonstrou 
valores mais baixos em relação aos domínios físico e ambiente, salientando-se, portanto, a necessidade de se reforçar as políticas de inclusão principalmente no que se refere a esses domínios.

O presente estudo demonstrou também a relação existente entre alguns aspectos sociais da vida da pessoa com deficiência como prática de atividade física e participação em atividades de lazer, com a percepção do seu nível de qualidade de vida.

Dessa forma, destaca-se a extrema importância a participação em atividades de lazer para melhora da qualidade de vida dessa população. Sendo um fator promotore de uma participação social ativa, se tornando uma importante ferramenta, uma vez que diversos estudos têm demonstrado os seus efeitos positivos das atividades de lazer sobre a promoção da saúde dessa população.

\section{REFERÊNCIAS}

ABNT- Associação Brasileira de Normas Técnicas. Nbr 9050: acessibilidade a edificações, mobiliário, espaços e equipamentos urbanos. 2. ed. 2004. Disponível em: $<$ http://www.mg.gov.br/sedh/ct/corde/dpdh/sicorde/principal/asp>. Acesso em: 07 mar. 2011.

BAMPI, L. N. da S.; GUILHEM, D.; LIMA, D. D. Qualidade de vida em pessoas com lesão medular traumática: um estudo com o WHOQOL-bref. Revista Brasileira de Epidemiologia. v. 11, n. 1,2008.

DIEHL, R. M. Jogando com as diferenças: jogos para crianças e jovens com deficiência. 2 ed. São Paulo: Phorte Editora, 2008. 215 p.

GORGATTI, M.G.; GORGATTI, T. O esporte para pessoas com deficiência. In: GORGATTI, M.G.; COSTA, R. F. Atividade física adaptada: qualidade de vida para pessoas com necessidades especiais. 2. ed. São Paulo: Manole, 2008. cap.15, p.532-570.

GUISELINI, M. Qualidade de vida: um Programa Prático para um corpo saudável. 2. ed. São Paulo: Gente, 1996.

HUNGER, D.; SQUARCINI, C. F. R.; PEREIRA, J. M. A pessoa portadora de deficiência física e o lazer. Revista Brasileira de Ciências do Esporte. Campinas, v. 25, n. 3,maio 2004. 
KINNEY, W. B.; COYLE, C. P. Predicting life satisfaction among adults with physicaldisabilities. Archives of physical medicine and rehabilitation. v. 73, p. 863-869,1992.

NOCE, F.; SIMIM, M. A. de M; MELLO, M. T. de. A percepção de qualidade de vida de pessoas portadoras de deficiência física pode ser influenciada pela prática de atividade física? Revista Brasileira de Medicina do Esporte. v. 15, n. 3, 2009.

OMS - ORGANIZAÇÃO MUNDIAL DE SAÚDE - DIVISÃO DE SAÚDE MENTAL GRUPO WHOQOL. Versão em português dos instrumentos de avaliação de qualidade de vida (WHOQOL). 1998. Disponível em: $<$ http://www.ufgs.br/psiq/whoqol.html $>$. Acesso em: 20 jan. 2010.

PEREIRA, R. J. Contribuição dos domínios físico, social, psicológico e ambiental para a qualidade de vida global de idosos. Revista de Psiquiatria do Rio Grande do Sul. v. 28, n.1, p. 27-38,jan./abr. 2006.

SHEPHARD, R.J. Benefits of sport and physical activity for the disabled: implications for the individual and for society. Scandinavian Journal of Rehabilitation Medicine. v. 23, p.51-59,1991.

THE WHOQOL GROUP. The World Health Organization quality of life assessment (WHOQOL): position paper from the World Health Organization. Social Science and Medicine.v. 41, p.1403-1410,1995.

ZUCHETO, Â. T.; CASTRO, R. L. V. G. de. As contribuições das atividades físicas para a qualidade de vida dos deficientes físicos. Revista Kinesis, Santa Maria, n. 26, p. $52-166$, maio 2002.

\section{Endereço das Autoras:}

Rejane Martins C. Lima

Maria Georgina Marques Tonello

Rua Guiana Inglesa, 450, apto 31

Bristol - Vila Mariana

Ribeirão Preto - SP - 14.075-210

Endereço Eletrônico: gina@ginatonello.com.br; rejane@unipam.edu.br;

priscillarqr@yahoo.com.br 